\title{
Chronic stress alters adrenal clock function in a sexually dimorphic manner
}

\author{
Matthew Stagl*, Mary Bozsik*, Christopher Karow*, David Wertz, lan Kloehn, Savin Pillai, Paul J Gasser, \\ Marieke R Gilmartin and Jennifer A Evans
}

Department of Biomedical Sciences, Marquette University, Milwaukee, Wisconsin, USA

Correspondence should be addressed to J A Evans: jennifer.evans@marquette.edu

*(M Stagl, M Bozsik and C Karow contributed equally to this work)

\begin{abstract}
Glucocorticoid production is gated at the molecular level by the circadian clock in the adrenal gland. Stress influences daily rhythms in behavior and physiology, but it remains unclear how stress affects the function of the adrenal clock itself. Here, we examine the influence of stress on adrenal clock function by tracking PERIOD2::LUCIFERASE (PER2::LUC) rhythms in vitro. Relative to non-stressed controls, adrenals from stressed mice displayed marked changes in PER2::LUC rhythms. Interestingly, the effect of stress on adrenal rhythms varied by sex and the type of stress experienced in vivo. To investigate the basis of sex differences in the adrenal response to stress, we next stimulated male and female adrenals in vitro with adrenocorticotropic hormone (ACTH). ACTH shifted phase and increased amplitude of adrenal PER2::LUC rhythms. Both phase and amplitude responses were larger in female adrenals than in male adrenals, an observation consistent with previously described sex differences in the physiological response to stress. Lastly, we reversed the sex difference in adrenal clock function using stress and sex hormone manipulations to test its role in driving adrenal responses to ACTH. We find that adrenal responsiveness to ACTH is inversely proportional to the amplitude of adrenal PER2::LUC rhythms. This suggests that larger ACTH responses from female adrenals may be driven by their lower amplitude molecular rhythms. Collectively, these results indicate a reciprocal relationship between stress and the adrenal clock, with stress influencing adrenal clock function and the state of the adrenal clock gating the response to stress in a sexually dimorphic manner.
\end{abstract}

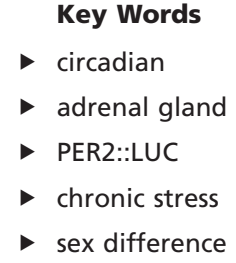

Journal of Molecular Endocrinology (2018) 60, 55-69

\section{Introduction}

Glucocorticoids are regulated in a dynamic manner over the course of the day, with elevated release at the start of the active phase of the circadian cycle. The daily increase in circulating glucocorticoids is mediated by rhythmic changes in the release of adrenocorticotrophic hormone (ACTH) and adrenal sensitivity to ACTH. Daily rhythms in both of these factors are driven by the circadian timekeeping system (Son et al. 2011, Nicolaides et al. 2017), which is a hierarchical collection of clocks located in tissues throughout the brain and body (Mohawk et al. 2012). The molecular mechanism driving cellular rhythms in tissues throughout the circadian system 
involves a 24-h transcriptional-translational negative feedback loop (Buhr \& Takahashi 2013) where Period (Per) and Cryptochrome (Cry) transcription is inhibited each day by their protein products. Genetic abrogation of molecular clock function eliminates glucocorticoid rhythms (Oster et al. 2006b, Son et al. 2008), which establishes that circadian mechanisms are essential for driving daily fluctuations in the activity of the hypothalamic-pituitaryadrenal axis (HPA).

The circadian system regulates the daily rhythm in glucocorticoid release via interactions among multiple tissue clocks (Son et al. 2011). At the top of the hierarchy, the master clock in the suprachiasmatic nucleus (SCN) maintains 24-h entrainment of glucocorticoid rhythms and induces light-induced glucocorticoid release by regulating endocrine and neural signals transmitted to the adrenal (Ishida et al. 2005, Oster et al. 2006b). In addition to top-down control, the adrenal gland itself exhibits intrinsic daily rhythms in glucocorticoid release, ACTH sensitivity and metabolic function in culture (Ungar \& Halberg 1962, Andrews \& Folk 1964). Intrinsic adrenal rhythms appear to be driven by a local molecular clock that gates transcriptional programs to regulate glucocorticoid biosynthesis and sensitivity to afferent input (Oster et al. 2006a,b, Son et al. 2008). Previous work has found that the intrinsic function of this adrenal clock differs markedly between males and females (Kuljis et al. 2013, Kloehn et al. 2016), which may contribute to sex differences in stress reactivity (Bangasser \& Valentino 2014, Goel et al. 2014). Understanding the precise role of the adrenal clock in driving stress responses and sex differences in its function remains important due to the pervasive influence of glucocorticoids on numerous physiological and behavioral processes, including metabolism, inflammation and memory function.

In addition to regulating daily glucocorticoid release, the circadian system itself can be influenced by glucocorticoid signaling (Kronfeld-Schor \& Einat 2012, Dickmeis et al. 2013). Previous work has established that glucocorticoids and stress can alter clock gene rhythms in many tissues (Oster et al. 2017) and that several clock genes contain glucocorticoid response elements in their promoters (Dickmeis et al. 2013). The reciprocal relationship between the circadian and glucocorticoid system suggest that stress may alter circadian function. Indeed, many patients with stress-related disorders display altered sleep and circadian rhythms (KronfeldSchor \& Einat 2012, Boland \& Ross 2015), with the most common consequence being a blunting of daily rhythms. Although many studies have assessed the effects of stress on daily rhythms, only a few studies have examined the effects of stress on the function of the adrenal clock itself (Bartlang et al. 2014, Razzoli et al. 2014, Tahara et al. 2015, Engeland et al. 2016). This issue warrants further study given the importance of the adrenal clock for gating glucocorticoid release and coordinating other tissue clocks in the circadian system (Balsalobre et al. 2000, Oishi et al. 2005, Segall et al. 2006, Reddy et al. 2007, Son et al. 2008, Kiessling et al. 2010, Pezuk et al. 2012). Thus, any effects of stress on the adrenal clock could produce pervasive changes in circadian timekeeping, homeostasis and susceptibility to stress-related disease.

Here, we investigate the influence of stress on the intrinsic function of the adrenal clock by tracking molecular rhythms using PERIOD2::LUCIFERASE (PER2::LUC) mice (Yoo et al. 2004). Based on previous reports of sex differences in adrenal clock function (Kuljis et al. 2013, Kloehn et al. 2016), we examined stress-induced changes in adrenal clock function in both male and female PER2::LUC mice. We find that exposure to stress produces marked changes in adrenal PER2::LUC rhythms, with the nature of the effect varying by type of stressor and sex. Additionally, we find sex differences in the intrinsic response of the adrenal clock to ACTH stimulation in vitro, which appear to be driven by the sexual diergic state of the adrenal clock. Thus, our work establishes that stress alters the function of the adrenal clock in a manner that differs by sex, which represents a novel bidirectional relationship between circadian function and glucocorticoids. These findings underscore the importance of considering the sexual dimorphic function of the adrenal clock when investigating mechanisms underlying sex differences in stress reactivity.

\section{Materials and methods}

\section{Mice}

Male and female homozygous PER2::LUC knockin mice (Yoo et al. 2004), backcrossed onto a C57BL/6 background, were bred and raised under a 24-h light:darkness cycle with $12 \mathrm{~h}$ of light and $12 \mathrm{~h}$ of darkness per day (LD12:12, lights-off: 1800 CST, which is defined as Zeitgeber time 12 (ZT12)). Throughout life, ambient temperature was maintained at $22 \pm 2^{\circ} \mathrm{C}$, and animals had ad libitum access to water and food (Teklad Rodent Diet \#8604). All procedures were conducted according to the NIH Guide for the Care and Use of Animals and were approved by 
the Institutional Animal Care and Use Committee at Marquette University.

\section{PER2::LUC tissue culture}

At 10-14 weeks of age, male and female PER2::LUC mice were anesthetized with isoflurane and killed by cervical dislocation 2-6h before the time of lights-off (i.e., ZT610). Adrenals were collected during late afternoon to minimize resetting effects of tissue culture (Davidson et al. 2009). Stress-induced changes in hormone levels were not assessed because the rising levels of corticosterone that occur at this time of day were expected to complicate comparisons across groups. However, previous work documents that stress procedures used here induce corticosterone release (e.g. Kelley et al. 2009, Jeong et al. 2013, Laukova et al. 2014). Adrenal glands were excised and placed in chilled Hank's Balanced Salt Solution. Adipose tissue was removed by hand with a scalpel, and adrenals were bisected before culture on a membrane with $1.2 \mathrm{~mL}$ of air-buffered Dulbecco's Modified Eagle's Medium (DMEM, Gibco 12100-046) containing $0.1 \mathrm{mM}$ beetle luciferin (Gold Biotechnologies). Bioluminescence rhythms were measured for 6-7 days with a luminometer (Actimetrics Inc., Evanston, IL, USA) inside a light-tight incubator set to $36^{\circ} \mathrm{C}$. Adrenal samples were weighed after 7 days of recording.

\section{Restraint stress}

To accommodate sex differences in body size, male and female PER2::LUC mice were physically restrained using clear disposable plastic restrainers (i.e., decapicones). Starting $6 \mathrm{~h}$ before lights-off (i.e. ZT6), mice were restrained for $2 \mathrm{~h}$ for either 1 day (acute restraint stress) or 7 consecutive days (chronic restraint stress). Previous studies have demonstrated that mice do not habituate to this protocol of repeated restraint stress (McQuade et al. 2006, Jeong et al. 2013). During restraint, mice were continuously monitored for body temperature, respiration and heart rate. Age-matched mice of each sex served as non-stressed controls. Control and stressed mice were weighed daily. Adrenal glands were cultured either immediately following restraint stress, $24 \mathrm{~h}$ later or 7 days after the last day of restraint stress.

\section{Simulated stress disorder}

Mice were exposed to a series of stress manipulations that models posttraumatic stress disorder (Wang et al. 2012).
This model involves exposure to five days of fear conditioning and a single day of prolonged stress. During fear conditioning, male and female PER2::LUC mice were transferred $4 \mathrm{~h}$ before lights-off (i.e., ZT8) into a conditioning chamber (Med Associates, Inc. Modular Test Chamber model ENV-008-FC, $30.5 \mathrm{~cm} \times 24.1 \mathrm{~cm} \times 21.0 \mathrm{~cm}$ ) housed within a soundattenuating box (Med Associates, Inc. Model ENV$022 \mathrm{MD}, \quad 63.5 \mathrm{~cm} \times 41.9 \mathrm{~cm} \times 39.4 \mathrm{~cm})$. After a 1 -min baseline period, a bright light was illuminated for $10 \mathrm{~s}$ and co-terminated with a $2 \mathrm{~s}, 1 \mathrm{~mA}$ scrambled foot shock provided by an electric shock circuit board (Med Associates, Inc. ENV-005-02A Rev. 1.0) and T/T Interface Cabinet Shock Generator (SG-6080C). After the shock, the mice remained in the chamber for $1 \mathrm{~min}$ before being returned to their home cage. The fear-conditioning procedure was repeated for five consecutive days. On the sixth day, mice were exposed to a day of single prolonged stress (i.e., 2-h restraint stress, 20-min forced swim stress in $24^{\circ} \mathrm{C}$ water and exposure to ether until loss of consciousness). Stressed mice and age-matched control mice were weighed daily. Mice were killed for in vitro culture of adrenal glands either $24 \mathrm{~h}$ or 7 days after the day of single prolonged stress.

\section{In vitro drug stimulation}

To directly investigate sex differences in the adrenal clock response to afferent input, adrenal samples from male and female PER2::LUC mice were stimulated in vitro with adrenocorticotropic hormone (ACTH, $100 \mathrm{nM}$ as in Yoder et al. 2014), dexamethasone (DEX, $10 \mu \mathrm{M}$ as in Pezuk et al. 2012) or acetylcholine (ACh, $10 \mu \mathrm{M}$ as in Boksa and Livett 1984). Adrenal samples were cultured for at least 3 days before drug administration at a specific phase of the PER2::LUC rhythm. The phase of drug administration was calculated relative to the time of peak PER2::LUC expression, which is defined here as circadian time 12 (CT12). Drugs were administered at one of six times spanning the circadian cycle (i.e., CT0, CT4, CT8, CT12, CT16, CT20). For drug administration, adrenal samples were removed from the luminometer and transferred to a biosafety cabinet on a heating pad set to $36^{\circ} \mathrm{C}$. Adrenal samples were stimulated directly with drugs diluted in vehicle (i.e., DMEM or DMSO) before dishes were resealed and returned to the luminometer. A subset of samples was pulsed with the inert vehicle to control for non-specific effects of this procedure. 


\section{Testosterone implantation}

To increase the amplitude of adrenal PER2::LUC rhythms, female mice received testosterone implants in vivo, as in study by Kloehn et al. (2016). Briefly, female mice were implanted at 12-14 weeks of age with a subcutaneous Silastic capsule (Dow Corning Corp, outer diameter $2.16 \mathrm{~mm}, 15 \mathrm{~mm}$ in length) filled with $10 \mathrm{~mm}$ of testosterone (Sigma, Cat\#T-1500) or left empty. Capsules were sealed with Silastic adhesive, washed in $70 \%$ ethanol and primed in sterile saline at $36^{\circ} \mathrm{C}$ overnight to prevent a bolus of hormone upon implantation. This procedure has been shown to effectively elevate testosterone to physiological levels for more than four weeks (Demas \& Nelson 1998, Rilianawati et al. 2000). Capsules were placed between the scapulae under isoflurane anesthesia, and the incision was closed with wound clips before treatment with nitrofurazone. Adrenal glands were collected four weeks later for PER2::LUC recording.

\section{Data analyses}

PER2::LUC rhythms were analyzed with Lumicycle analysis software (Actimetrics Inc.) by fitting a damped sine wave to the detrended time series (i.e., $24 \mathrm{~h}$ running average subtracted from the raw data) starting with the time of the first trough in vitro. For each sample, the period, damping rate and goodness of fit were recorded from the sine wave calculated by Lumicycle (Table 1). Also, the time of daily peaks and troughs were recorded for each cycle in vitro, from which we calculated phase and precision (i.e., the inverse of the s.D. of cycle-to-cycle period length). Lastly, the baseline-subtracted time series were exported into Excel to calculate the amplitude of the PER2::LUC rhythm (difference between peak and trough expression) on each cycle in vitro.

To analyze adrenal response to in vitro stimulation, the first three cycles were used to project the time of drug application. The projected time of drug application was determined by adding the required number of circadian hours to the time of the 3 rd peak in vitro (e.g., CT18 $=$ CT12 +6 * $(24 /$ period length $)$. Drug-induced phase shifts were calculated for each sample using the difference between the actual and predicted peak time on the subsequent cycle in vitro. To calculate the prediction error of this analytical method, first we calculated the difference between the predicted and actual time for the 3rd trough in vitro, which occurred before drug administration. The prediction error for this validation method was very low $(0.29 \pm 0.07 \mathrm{~h})$ and did not differ by sex (males: $0.36 \pm 0.13 \mathrm{~h}$, females: $0.23 \pm 0.06 \mathrm{~h}$,

Table 1 Effects of chronic and acute stress on the adrenal clock.

\begin{tabular}{|c|c|c|c|c|c|c|}
\hline & \multicolumn{2}{|c|}{ Control } & \multicolumn{2}{|c|}{ Stress } & \multicolumn{2}{|c|}{ Recovery } \\
\hline & Female & Male & Female & Male & Female & Male \\
\hline \multicolumn{7}{|l|}{ Chronic restraint stress } \\
\hline$n$ & 14 & 15 & 14 & 16 & 9 & 12 \\
\hline Adrenal weight (mg) & $1.7 \pm 0.1$ & $1.1 \pm 0.1+$ & $1.6 \pm 0.1$ & $1.4 \pm 0.1 *$ & $1.4 \pm 0.1 *$ & $1.2 \pm 0.1$ \\
\hline Peak time (h) & $13.8 \pm 0.4$ & $15.2 \pm 0.4+$ & $11.9 \pm 0.7 *$ & $10.4 \pm 0.7 *+$ & $14.1 \pm 0.4$ & $14.3 \pm 0.9$ \\
\hline Period (h) & $23.0 \pm 0.2$ & $22.9 \pm 0.2$ & $26.0 \pm 0.8^{*}$ & $24.2 \pm 0.3^{*}$ & $26.8 \pm 0.4^{*}$ & $23.1 \pm 0.2+$ \\
\hline Precision (1/h) & $0.8 \pm 0.1$ & $2.1 \pm 0.3+$ & $0.5 \pm 0.1 *$ & $0.8 \pm 0.1 *+$ & $0.4 \pm 0.1 *$ & $1.0 \pm 0.3^{*}$ \\
\hline Damping (days) & $1.2 \pm 0.1$ & $1.6 \pm 0.1+$ & $1.0 \pm 0.1 *$ & $0.9 \pm 0.1 *$ & $1.0 \pm 0.1 *$ & $1.4 \pm 0.1+$ \\
\hline \multicolumn{7}{|l|}{ Acute restraint stress } \\
\hline$n$ & 8 & 8 & 8 & 8 & 8 & 8 \\
\hline Adrenal weight (mg) & $1.5 \pm 0.1$ & $1.1 \pm 0.1+$ & $2.1 \pm 0.1 *$ & $1.6 \pm 0.1^{*}+$ & $1.7 \pm 0.2$ & $1.2 \pm 0.2+$ \\
\hline Peak time (h) & $13.4 \pm 0.8$ & $14.9 \pm 0.7$ & $14.7 \pm 0.9$ & $13.6 \pm 0.5^{*}$ & $14.5 \pm 0.5$ & $15.7 \pm 0.6$ \\
\hline Period (h) & $22.8 \pm 0.2$ & $22.6 \pm 0.1$ & $22.7 \pm 0.2$ & $22.7 \pm 0.1$ & $22.6 \pm 0.3$ & $23.0 \pm 0.2$ \\
\hline Precision & $0.7 \pm 0.1$ & $1.6 \pm 0.3+$ & $0.7 \pm 0.1$ & $1.6 \pm 0.2+$ & $0.8 \pm 0.2$ & $0.9 \pm 0.1$ \\
\hline Damping (days) & $1.2 \pm 0.1$ & $1.6 \pm 0.1+$ & $1.4 \pm 0.1$ & $1.6 \pm 0.1$ & $1.3 \pm 0.1$ & $1.4 \pm 0.1$ \\
\hline \multicolumn{7}{|l|}{ Chronic stress model } \\
\hline$n$ & 8 & 8 & 8 & 8 & 8 & 8 \\
\hline Adrenal weight (mg) & $1.6 \pm 0.1$ & $1.2 \pm 0.1+$ & $1.6 \pm 0.1$ & $1.2 \pm 0.1+$ & $1.5 \pm 0.1$ & $1.1 \pm 0.1+$ \\
\hline Peak time (h) & $14.8 \pm 1.6$ & $15.3 \pm 0.8$ & $17.7 \pm 0.3$ & $15.7 \pm 0.4+$ & $14.6 \pm 0.9$ & $15.4 \pm 0.3$ \\
\hline Period (h) & $23.0 \pm 0.3$ & $22.6 \pm 0.1$ & $22.5 \pm 0.2$ & $22.5 \pm 0.1$ & $23.4 \pm 0.4$ & $22.8 \pm 0.1$ \\
\hline Precision & $0.4 \pm 0.1$ & $1.3 \pm 0.2+$ & $1.1 \pm 0.2 *$ & $2.1 \pm 0.3^{*}+$ & $0.7 \pm 0.1 *$ & $1.2 \pm 0.2+$ \\
\hline Damping (days) & $1.3 \pm 0.1$ & $2.0 \pm 0.1+$ & $1.4 \pm 0.1$ & $1.9 \pm 0.1+$ & $1.5 \pm 0.1$ & $1.9 \pm 0.1+$ \\
\hline
\end{tabular}

Peak time expressed as Zeitgeber time (ZT), lights-off $=$ ZT12.

*Differs from same-sex control, $P<0.05 ;$ + differs by sex within group, $P<0.05$. 
$t(125)=0.94, P>0.3)$. Also, we calculated the prediction error using an independent set of unstimulated samples by determining the difference between the predicted and actual peak time on the 5th cycle in vitro. Again, the prediction error was low $(0.55 \pm 0.45 \mathrm{~h})$ and did not differ by sex (males: $0.53 \pm 0.88 \mathrm{~h}$, females: $0.57 \pm 0.3 \mathrm{~h}$, $t(14)=0.05, P>0.9)$. Collectively, these results indicate that drug-induced phase shifts $>0.5 \mathrm{~h}$ should be detectable, demonstrate the validity of predicting circadian phase using these methods and establish that this analytical approach can be applied with equal confidence to samples from both sexes.

Video records of fear-conditioning trials and forced swim exposure were scored off-line. Freezing was used as the measure of fear during fear conditioning and was defined as the cessation of all movement except that required for respiration, as in the study by Dellapolla et al. (2017). The behavior of each mouse was scored as freezing or not freezing once every $4 \mathrm{~s}$ by trained observers blind to the sex of the mouse. The inter-rater reliability coefficient was 0.94. Freezing was expressed as the percent of time spent freezing during each minute of the conditioning session, and acquisition of fear conditioning was quantified by assessing percent freezing during the first minute of the trial each day. During forced swim, we recorded the amount of time spent engaging in active escape behaviors (mobility) and passive behavior (immobility). Mobility was defined as any movements other than those required for floating.

Statistical analyses were performed with JMP software (SAS Institute, Cary, NC, USA). Body weight, adrenal weight, time of peak PER2::LUC expression on the 1st cycle in vitro, period length, damping and precision of PER2::LUC rhythms were analyzed with a full factorial ANOVA (factors: stress, sex, stress * sex). Group differences relative to control were assessed using Dunnett's test. Cycle-to-cycle changes in amplitude were analyzed with a full factorial repeated-measures ANOVA (factors: stress, sex, time, stress *sex, stress*time, sex *time, stress ${ }^{*}$ sex*time). Group differences were assessed with a repeated-measures ANOVA divided by the stress group or sex, followed by full factorial ANOVA and post hoc tests using least square means (LSM) contrasts with Bonferroni correction $(\alpha=0.01)$. Sex differences in conditioned fear acquisition and forced swim immobility were analyzed using a repeated-measures ANOVA and Student's $t$ test, respectively. Data are represented in figures and tables as mean \pm S.E.M.

\section{Results}

\section{Chronic restraint stress suppresses adrenal clock function}

Male and female PER2::LUC mice were exposed to 2-h restraint for 7 consecutive days (Fig. 1A). Chronic restraint stress reduced body weight gain in both sexes $(P<0.05)$, consistent with the stressful nature of this procedure (McQuade et al. 2006, Jeong et al. 2013). Further, chronic restraint stress influenced adrenal weight in a manner that differed by sex (Table 1). Specifically, non-stressed females had larger adrenal samples than non-stressed males $(P<0.05)$, and chronic restraint stress eliminated this sex difference by increasing adrenal weight in males (Table 1). These data indicate that chronic restraint produced physiological stress.

To test whether chronic restraint stress influenced adrenal clock function, adrenals were collected either 1 day or 7 days after the last exposure to restraint (Fig. 1A). Adrenals from non-stressed males displayed larger amplitude PER2::LUC than those from non-stressed females (Fig. 1B and C, sex *time: $P<0.0001$ ), consistent with previous work (Kuljis et al. 2013, Kloehn et al. 2016). Chronic restraint attenuated PER2::LUC rhythms in both male and female adrenals and eliminated the sex difference in rhythm amplitude (Fig. 1B and D, sex ${ }^{*}$ time: $P>0.4$ ). The loss of the sex difference was largely due to a marked decrease in the amplitude of adrenal rhythms in samples from stressed males (Fig. 1D, $P<0.01$ ), although female adrenals also displayed a modest decrease in amplitude (Fig. 1D, $P<0.01)$. One week without restraint stress was sufficient to restore the sex difference in PER2::LUC amplitude (Fig. 1B and $C$, sex time: $P<0.0001$ ), but recovery male and female adrenals continued to display lower amplitude rhythms than non-stressed controls (Fig. 1D, $P<0.01$ ). In addition, chronic restraint stress in both sexes advanced the time of peak PER2::LUC expression on the first cycle in vitro, lengthened period, decreased precision and accelerated damping over time (Table 1). Overall, effects on phase and amplitude were larger in males, but changes in period and damping were longer lasting in females because they persisted for 7 days. Collectively, these results demonstrate that chronic restraint stress influences the intrinsic function of the adrenal clock, with differential effects in males and females.

Next, we tested whether a single exposure to restraint stress would influence adrenal clock function (Fig. 2A). Again, a sex difference in adrenal amplitude was evident in 

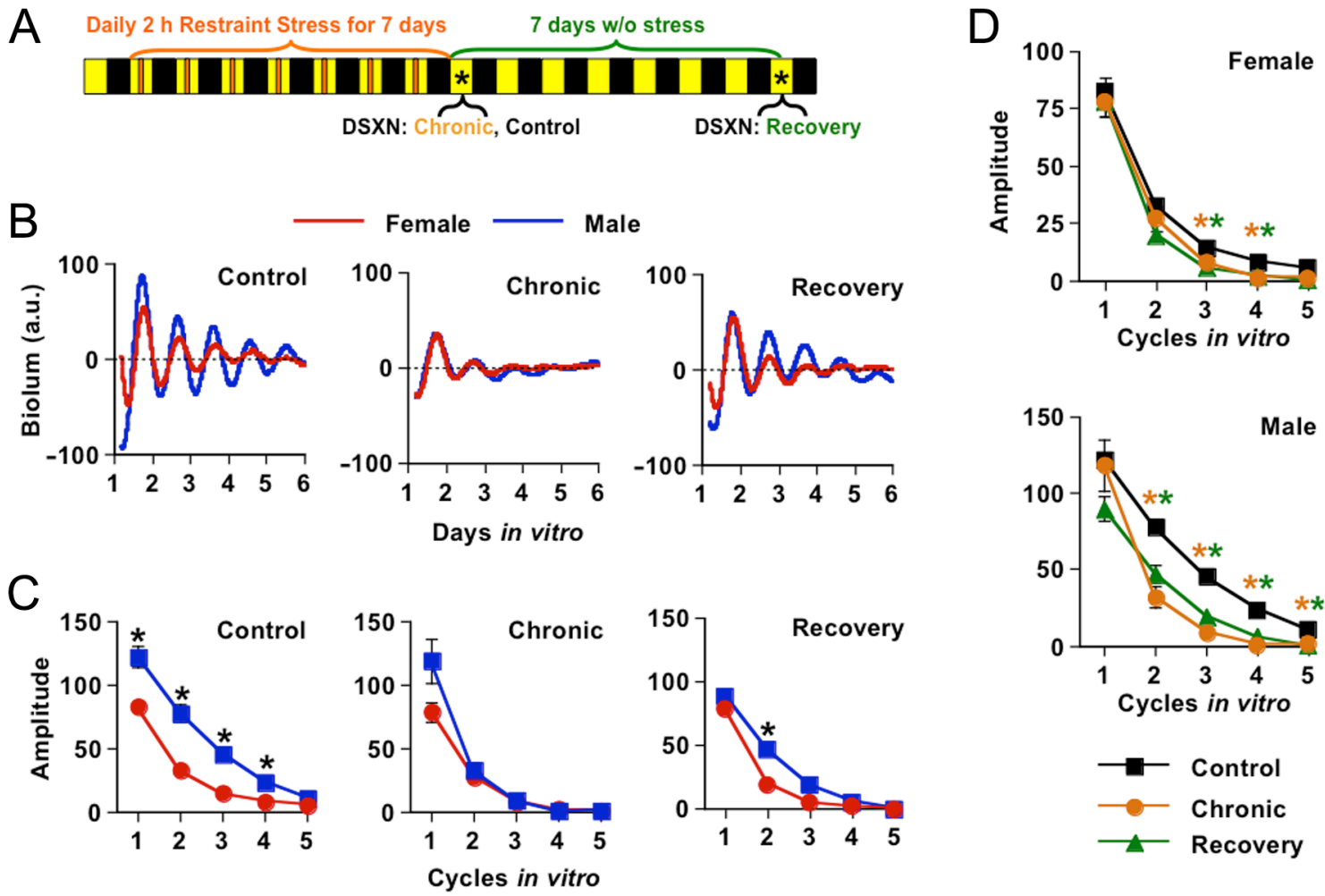

Figure 1

Chronic restraint stress influences adrenal clock function. (A) Schematic illustrating in vivo chronic restraint stress protocol and adrenal collection for PER2::LUC recording. Exposure to restraint stress for 7 days decreased the gain in body weight in both sexes (stress:: $F(1,18) 22.8, P<0.0005 ;$ sex: $F(1,18)=0.05, P>0.8$; stress * sex: $F(1,18)=0.6, P>0.5)$. Adrenals were collected from non-stressed controls (Control) and stressed mice either 1 day (Chronic) or 7 days (Recovery) after the last day of restraint. *DSXN: day and time of dissection for indicated group. (B) Representative PER2::LUC time series from male and female adrenals from the non-stressed control, chronic stress and recovery groups. (C) Chronic restraint stress eliminated the sex difference in the amplitude of adrenal PER2::LUC rhythms (repeated-measures ANOVA, non-stressed controls: sex: $F(1,27)=39.7, P<0.0001$; time: $F(4,24)=109.0, P<0.0001$; sex * time: $F(4,24)=12.05, P<0.0001$; chronic: sex: $F(1,28)=2.5, P>0.1$; time: $F(4,25)=25.7, P<0.0001$; sex time: $F(4,25)=1.0$, $P>0.4$; recovery: sex: $F(1,19)=10.1, P<0.0001$; time: $F(4,16)=41.1, P<0.0001$; sex *time: $F(4,16)=10.3, P<0.0001)$. (D) Chronic restraint stress reduced the amplitude of PER2::LUC rhythms in males and females (repeated-measures ANOVA, males: stress: $F(2,40)=11.6, P<0.0001 ;$ time: $F(4,37)=67.8, P<0.0001$; stress * time: $F(8,74)=4.2, P<0.0001$; females: stress: $F(2,34)=2.9, P=0.07$; time: $F(4,31)=90.8, P<0.0001$; stress * time: $F(8,62)=3.1, P<0.01)$. Note that data in (D) are re-plotted from (C) to facilitate visualization of effects of chronic stress in each sex. Number of cultures per group $=9-16$ (Table 1). *LSM contrasts, $P<0.01$, color-coded in (D) to represent comparisons between each stress group and non-stressed controls.

non-stressed controls (Fig. $2 \mathrm{~B}$ and $\mathrm{C}$, sex*time: $P<0.005$ ), but this was not eliminated by acute restraint stress (Fig. 2C, sex * time: $P<0.01)$. Unlike chronic stress, acute stress did not significantly influence PER2::LUC amplitude when assessed separately for either sex (Fig. 2D). Likewise, other rhythmic parameters were not altered, with the exception that adrenals from stressed males displayed a small phase advance when collected immediately after restraint (Table 1 ). Thus, most effects of restraint stress on adrenal clock function require multiple days of exposure to this procedure.

\section{Sex difference in adrenal response to a model of posttraumatic stress disorder}

Finding that chronic restraint stress altered adrenal clock function, we next wanted to assess sex differences in the adrenal response to a chronic stress procedure developed as a mouse model of posttraumatic stress disorder (Wang et al. 2012, Daskalakis et al. 2013). This model involves five days of fear conditioning (FC) and a single day of prolonged stress (SPS, Fig. 3A). During the fear-conditioning phase of this procedure, both male and female mice displayed an increase in fear behavior over consecutive days, with high levels of freezing on the last day of training. No sex differences were evident in the pattern of increased fear behavior across days of conditioning (Fig. 3B, $P>0.5$ ). Further, male and female mice did not differ in the time spent immobile during forced swim on the day of SPS (Fig. 3C, $P>0.6$ ). Lastly, changes in body weight during exposure to $\mathrm{FC}+$ SPS stress did not vary by sex $(P>0.3)$. These data indicate that male and female mice displayed similar overt responses to the FC+SPS stress procedure. 
A

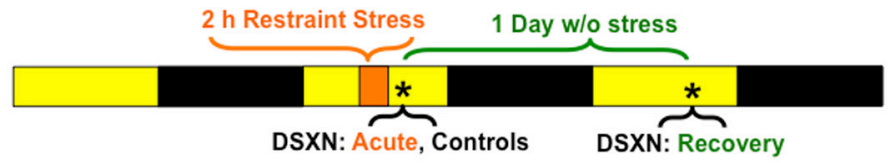

B
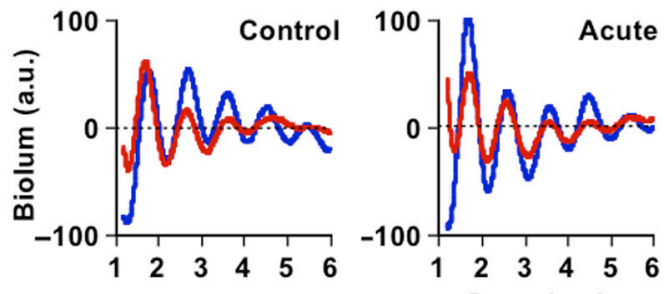

Days in vitro

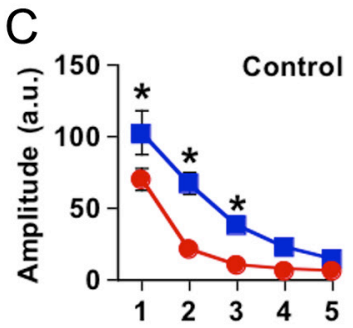

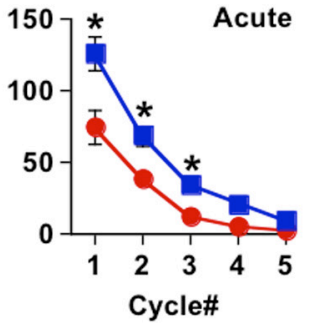
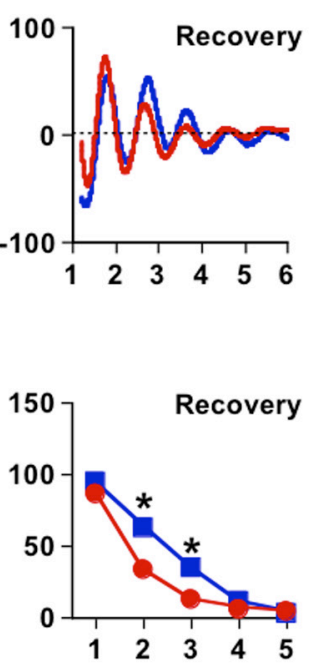
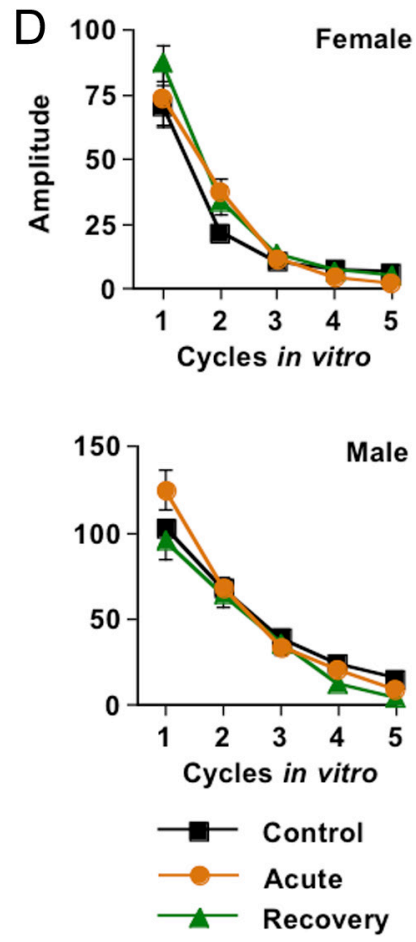

Figure 2

A single exposure to 2-h restraint stress does not influence adrenal clock function. (A) Schematic illustrating in vivo acute restraint stress protocol and adrenal collection for PER2::LUC recording. Adrenals were collected from non-stressed controls (Control) and stressed mice either immediately after the 2-h restraint (Acute) or the next day (Recovery). *DSXN: day and time of dissection for indicated group. (B) Representative PER2::LUC time series from male and female adrenals from the non-stressed control, acute stress and recovery groups. (C) Acute restraint stress did not eliminate the sex difference in adrenal amplitude (repeated-measures ANOVA, non-stressed controls: sex: $F(1,14)=16.6, P<0.005$; time: $F(4,11)=20.89, P<0.0001$; sex * time:

$F(4,11)=5.18, P<0.05$; acute: sex: $F(1,14)=22.0, P<0.0005$; time: $F(4,11)=29.6, P<0.0001$; sex $*$ time: $F(4,11)=5.76, P<0.05$; recovery: sex: $F(1,14)=3.87$, $P=0.07$; time: $F(4,11)=25.5, P<0.0001$; sex * time: $F(4,11)=5.9, P<0.01)$. (D) Acute restraint stress did not reduce the amplitude of PER2::LUC rhythms in males or females (repeated-measures ANOVA, males: stress: $F(2,21)=0.8, P>0.4$; time: $F(4,18)=49.7, P<0.0001$; stress * time: $F(8,36)=1.4, P>0.2 ;$ females: stress: $F(2,21)=0.9, P>0.4$; time: $F(4,18)=27.8, P<0.0001$; stress * time: $F(8,36)=2.1, P=0.06)$. Note that data in (D) are re-plotted from (C) to illustrate the lack of effect of acute stress in both sexes. Number of cultures per group $=8$. * LSM contrasts, $P<0.01$.

Despite similar overt responses, male and female adrenals displayed a marked sex difference in the response of the adrenal clock (Fig. 3D and E). Female adrenals, but not male adrenals, displayed an increase in the amplitude of PER2::LUC rhythms after exposure to FC+SPS stress (Fig. 3D and E, females: stress *time: $P<0.0005$; males: stress*time: $P>0.1$ ). Moreover, the increase in rhythm amplitude of female adrenals persisted for at least 7 days following FC+SPS (Fig. 3D and E). FC+SPS increased the amplitude of PER2::LUC rhythms in female adrenals, but the sex difference in amplitude was not eliminated by this procedure (Fig. 3F, sex *time: $P<0.05)$. Interestingly, rhythm precision was increased by FC+SPS in both sexes, and this effect was longer lasting in female adrenals (Table 1). These results reveal that FC+SPS increased amplitude and precision of adrenal rhythms, with stronger and longer-lasting effects in females. These results are opposite from those found for chronic restraint stress, which decreased the amplitude and precision of molecular rhythms in adrenals of both sexes (c.f., Fig. 1D).

\section{Sex differences in adrenal clock responsiveness to $\mathrm{ACTH}$}

Based on previous research (Kitay 1961a, Yoder et al. 2014), we hypothesized that sexually dimorphic responses to in vivo stressors may be driven by differences in the response of the adrenal clock to ACTH stimulation. To test this hypothesis, we directly examined in vitro responses of male and female adrenals to ACTH application (Fig. 4A). Adrenal samples were collected from non-stressed mice and treated with ACTH at specific phases of the PER2::LUC rhythm to measure drug-induced changes in adrenal clock function. As expected, ACTH reset the phase of adrenal PER2::LUC 
A

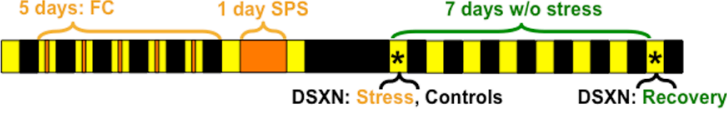

B

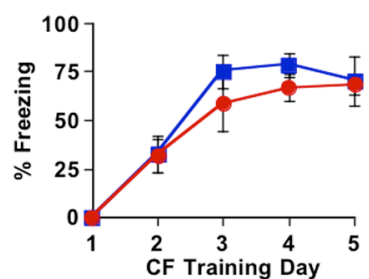

C

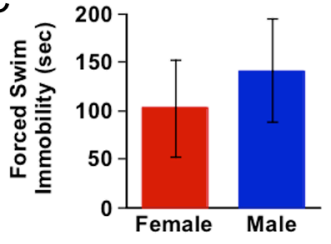

D

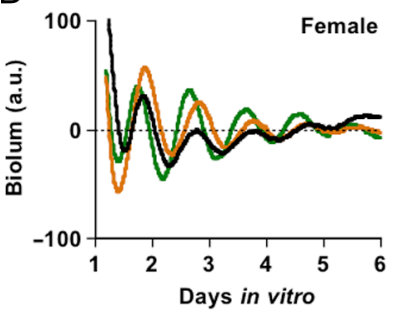

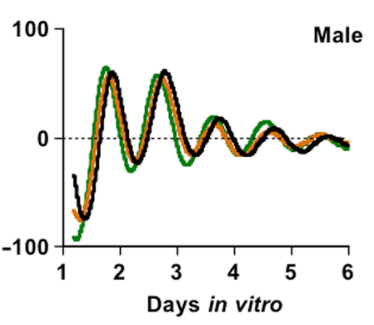

E
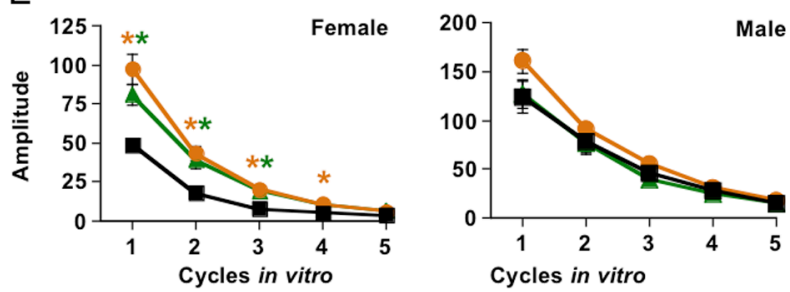

F

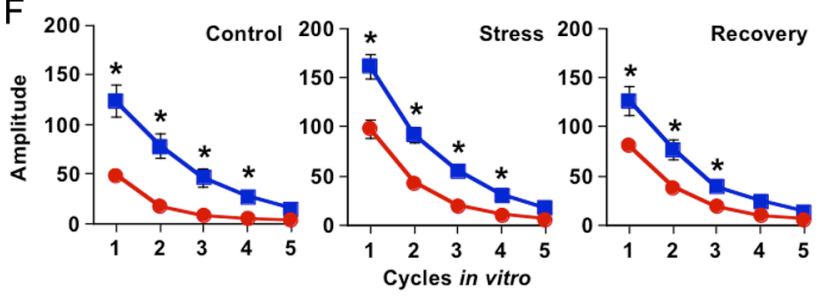

Figure 3

Sex differences in the response of the adrenal clock to a chronic stress model due to increased amplitude of PER2::LUC rhythms in female adrenals. (A) Schematic illustrating in vivo chronic stress protocol and adrenal collection for PER2::LUC recording. Adrenals were collected from non-stressed controls (Control) and stressed mice either 1 day (Stress) or 7 days (Recovery) after single prolonged stress. *DSXN: day and time of dissection for indicated group. (B) Male and female mice displayed similar fear behavior over consecutive days of FC training. (C) Male and female mice displayed similar duration of immobility during forced swim on the day of SPS. (D) Representative PER2::LUC time series from male and female adrenals collected from non-stressed control, stress, and recovery mice. (E) Stress increased PER2::LUC amplitude in female adrenals (repeated-measures ANOVA stress: $F(2,21)=12.4$, $P<0.0005$; time: $F(4,18)=95.5, P<0.0001$; stress * time: $F(8,36)=5.1, P<0.0005)$, but did not influence PER2::LUC amplitude in males (repeated-measures ANOVA, males: stress: $F(2,21)=1.2, P>0.3$; time: $F(4,18)=69.7, P<0.0001$; stress * time: $F(8,36)=1.6, P>0.1)$. (F) Exposure to the stress manipulation did not eliminate sex differences in the amplitude of PER2::LUC rhythms in any group (repeated-measures ANOVA, non-stressed controls: sex: $F(1,14)=19.8$, $P<0.001$; time: $F(4,11)=45.5, P<0.0001$; sex *time: $F(4,11)=7.2, P<0.005$; stress: sex: $F(1,14)=28.1, P<0.0005 ;$ time: $F(4,11)=56.1, P<0.0001 ;$ sex $*$ time: $F(4,11)=4.8, P<0.05$; recovery: sex: $F(1,14)=10.7, P<0.01$; time: $F(4,11)=38.1, P<0.0001$; sex $*$ time: $F(4,11)=2.8, P=0.08)$. Number of cultures per group $=8$. *LSM contrasts, $P<0.01$, color-coded in $(E)$ to represent comparisons between each stress group and non-stressed controls.

rhythms in males (Fig. 4A and B), with the magnitude and direction of phase shifts similar to those found in previous work (Yoder et al. 2014). Relative to vehicletreated controls, ACTH shifted the rhythm later when applied at CT8, CT12, CT16 and CT20 (i.e., phase delays during late subjective day and subjective night), but shifted the rhythm earlier when applied at CT0 and CT4 (i.e., phase advances during early subjective day). In addition, ACTH increased the amplitude of PER2::LUC rhythms on the cycle following treatment in a manner that varied by the time of stimulation (Fig. 4C). The magnitude of both the ACTH-induced resetting response and the ACTH-induced amplitude potentiation response was negatively correlated with the amplitude of the PER2::LUC rhythm on the cycle prior to treatment (resetting $-r=-0.37, P<0.01$; potentiation $-r=-0.26$, $P<0.05)$. This indicates that larger resetting and amplitude responses were elicited from adrenal samples with lower PER2::LUC amplitude prior to the pulse. Further, phase resetting and amplitude potentiation were positively correlated $(r=0.52, P<0001)$ because phase delays were associated with smaller amplitude changes than phase advances (i.e., negative phase shifts were associated with small changes in amplitude, while positive phase shifts were associated with large changes in amplitude).

Consistent with the inverse relationship between adrenal PER2::LUC amplitude and ACTH responsiveness, there was a striking sex difference in the magnitude of both ACTH-induced effects (Fig. 4D). Female adrenals displayed larger resetting and amplitude responses than male adrenals at every phase tested (sex * time: $P<0.0001)$. As in male adrenals, there was an inverse relationship between PER2::LUC amplitude prior to the pulse and the magnitude of ACTH-induced resetting and potentiation response in female adrenals (resetting $-r=-0.49, P<0.001$; potentiation $-r=-0.41, P<0.01)$, and these two responses were positively correlated $(r=0.7, P<0.0001)$. Importantly, there was no sex difference in the response to vehicle treatment (Fig. 4B and C, sex*time: $P>0.4$ ), and phase 


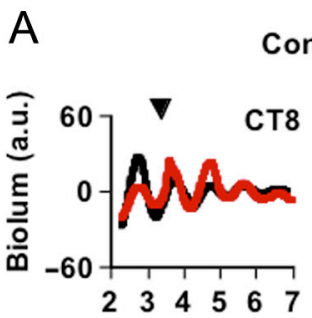

Control

Female ACTH
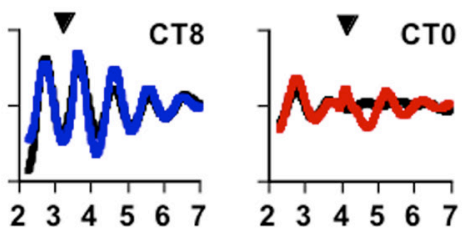

Male ACTH

Days in vitro

B $\longrightarrow$ -

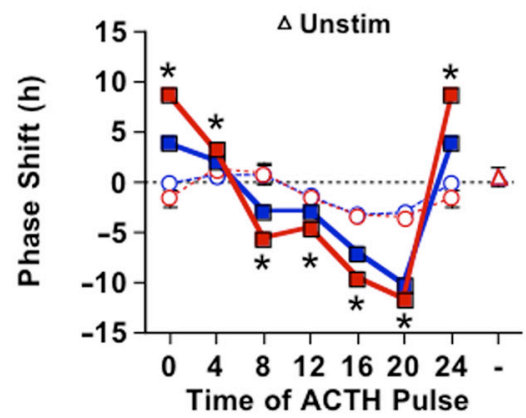

C

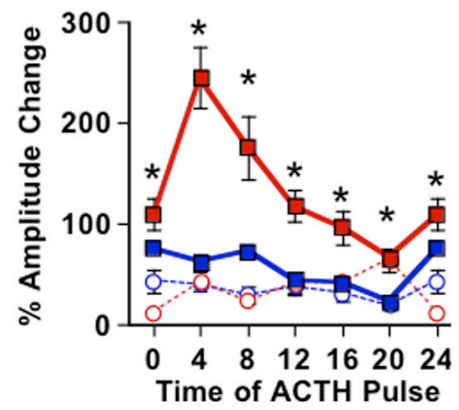

D

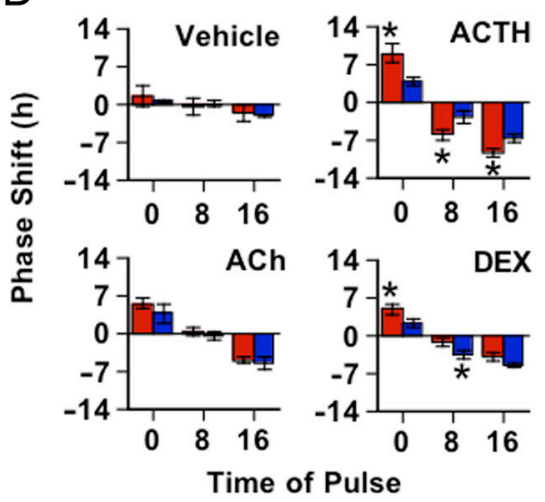

E

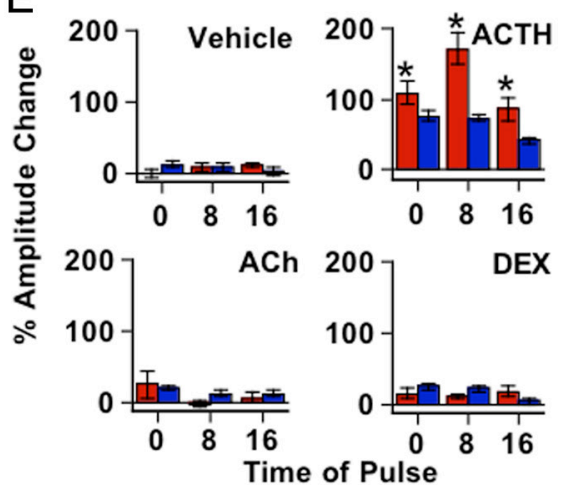

Figure 4

Sex differences in the intrinsic response of the adrenal clock to ACTH. (A) Representative PER2::LUC time series from male and female adrenals treated with ACTH at CT 0 or CT8. Each ACTH-treated sample is plotted along with a same-sex vehicle-treated control (black trace). Arrowhead indicates time of treatment. (B) Phase resetting responses of male and female adrenals treated with ACTH or vehicle. Male and females adrenals differed in their response to ACTH treatment (sex: $F(1,151)=4.43, P<0.05$, time: $F(5,151)=405.5, P<0.0001$, sex * time: $F(5,151)=19.2, P<0.0001)$, but not vehicle treatment (sex: $F(1,146)=1.2, P>0.2$, time: $F(5,146)=18.4, P<0.0001$, sex *time: $F(5,146)=0.9, P>0.5)$. Note that the response of unstimulated samples did not differ by sex (data plotted at time ' $-{ }^{\prime}, t(1,14)=-0.05, P>0.9$, see 'Methods' section for more information). Number of cultures per group $=8-12 / g r o u p$. (C) ACTH-induced amplitude potentiation was larger in female adrenals than male adrenals $($ sex: $F(1,151)=132.6, P<0.0001$, time: $F(5,151)=21.6, P<0.0001$, sex * time: $F(5,151)=9.8, P<0.0001)$. (D) ACTH induces a drug-specific pattern of sex differences in resetting that was not observed with acetylcholine (ACh), dexamethasone (DEX) or vehicle treatment (ACTH: sex: $F(1,18)=0.1, P>0.7$, time: $F(2,18)=139.2, P<0.0001$, sex $*$ time: $F(2,18)=13.3, P<0.0005$; ACh: sex: $F(1,23)=1.5, P>0.2$, time: $F(2,23)=42.0, P<0.0001$, sex *time: $F(2,23)=0.2, P>0.8$; $D E X:$ sex: $F(1,22)=10.2, P<0.005$, time: $F(2,22)=60.0$, $P<0.0001$, sex * time: $F(2,22)=0.3, P>0.7$; vehicle: sex: $F(1,19)=1.4, P>0.2$, time: $F(2,19)=8.5, P<0.005$, sex $*$ time: $F(2,19)=1.02, P>0.3)$. $(E)$ Adrenal amplitude was increased by $\mathrm{ACTH}$, but not by $\mathrm{ACh}$, DEX or vehicle $(\mathrm{ACTH}$ : sex: $F(1,18)=27.7, P<0.0001$, time: $F(2,18)=9.1, P<0.005$, sex $*$ time: $F(2,18)=3.2, P=0.06$; ACh: sex: $F(1,23)=0.5, P>0.4$, time: $F(2,23)=2.2, P>0.1$, sex * time: $F(2,23)=0.5, P>0.8$; $D E X:$ sex: $F(1,22)=0.2, P>0.6$, time: $F(2,22)=1.2, P>0.3$, sex * time: $F(2,22)=2.4 P>0.1$; vehicle: sex: $F(1,19)=0.03, P>0.8$, time: $F(2,19)=0.4, P>0.6$, sex $*$ time: $F(2,19)=0.3, P>0.3)$. LSM contrasts, ACTH-stimulated male vs female, $P<0.01$.

projections for unstimulated controls did not differ by sex (Fig. 4B, P>0.9). These results indicate that the phase of female adrenal rhythms is not inherently less stable or harder to predict. Further, greater overall responses in female adrenals were specific to ACTH, because responses to acetylcholine or dexamethasone were not consistently larger in female adrenals (Fig. 4D and E). These results reveal that there is a large sex difference in the ACTH responsiveness of the adrenal clock, which is related to the sexually dimorphic state of the molecular clock mechanism.

\section{Adrenal clock responsiveness to ACTH is inversely proportional to rhythm amplitude}

Based on the finding that PER2::LUC amplitude and ACTH responsiveness are negatively correlated, we sought to test whether rhythm amplitude drives the magnitude of ACTH responses. We predicted that if this were the case and the sex difference in adrenal amplitude were to be reversed, male adrenals would display a greater ACTH response than female adrenals (and vice versa). To achieve this reversal, we decreased adrenal amplitude in 
A
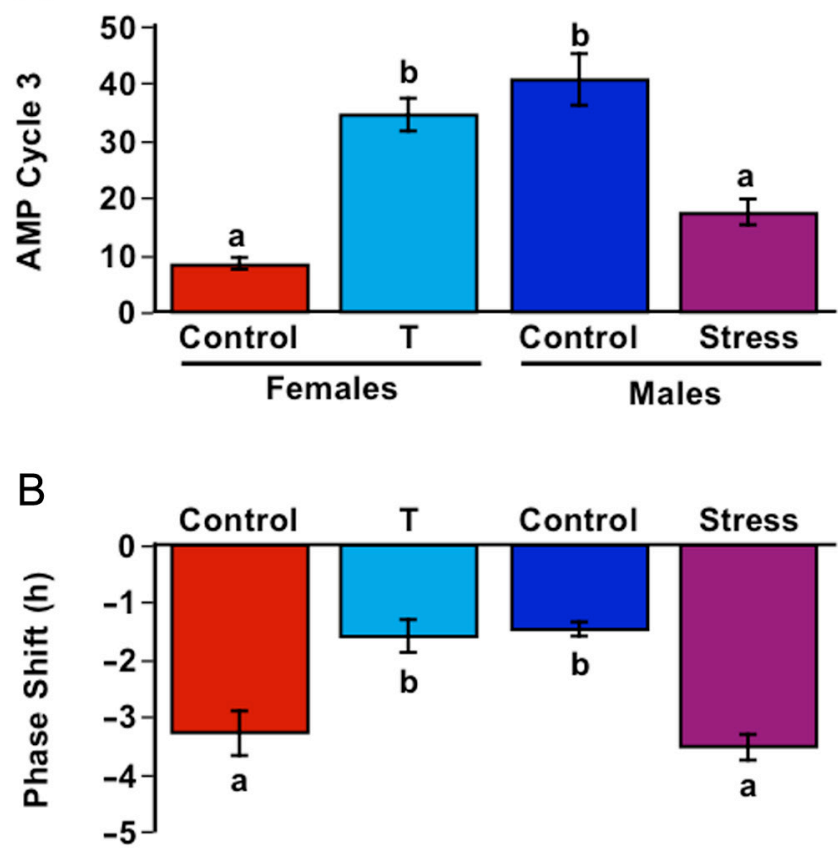

C

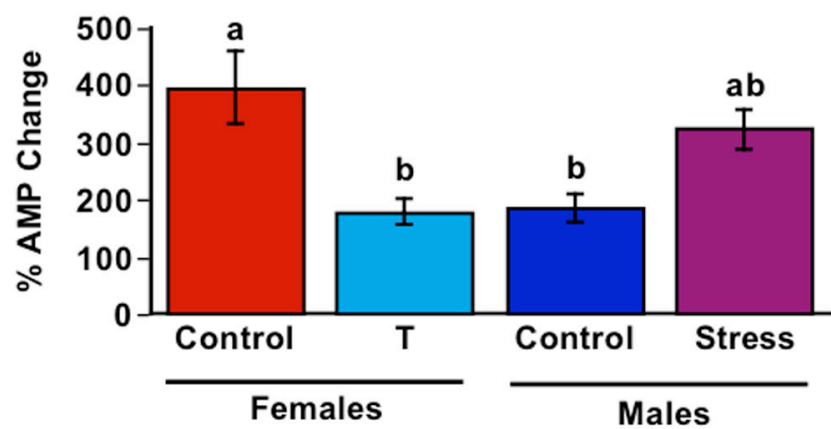

Figure 5

Reversing the sex difference in adrenal clock amplitude inverted the dimorphism in ACTH responsiveness. (A) Testosterone (T) administration to females in vivo increased the amplitude (AMP) of the PER2::LUC rhythm on the third cycle in vitro, whereas 7 days of chronic restraint stress in vivo decreased amplitude in male adrenals on the third cycle in vitro (sex: $F(1,82)=7.5, P<0.01$; group: $F(1,82)=0.2, P>0.6$; sex * group: $F(1,82)=77.2, P<0.0001)$. (B) Regardless of sex, adrenals with low amplitude displayed higher resetting responses when pulsed with ACTH at CT08 on the fourth cycle in vitro (sex: $F(1,36)=0.04, P>0.8$; group: $F(1,36)=0.5, P>0.4$; sex ${ }^{*}$ group: $\left.F(1,36)=52.4, P<0.0001\right)$. (C) Adrenals with low amplitude displayed higher amplitude responses when pulsed with ACTH at CT08 on the fourth cycle in vitro (sex: $F(1,36)=0.6, P>0.4$; group: $F(1,36)=0.9, P>0.3$; sex group: $F(1,36)=19.1, P=0.0001)$. Groups that do not share letters in common differ significantly, Tukey's HSD, $P<0.05$.

males using 7 days of restraint stress in vivo and increased adrenal amplitude in females using testosterone treatment in vivo (Kloehn et al. 2016). As expected, males that received 7 days of chronic restraint stress had adrenals with lower amplitude PER2::LUC rhythms prior to the ACTH pulse (Fig. 5A). Adrenal samples were stimulated with ACTH on the 4th cycle in vitro at CT08, which was a phase associated with larger responses in female adrenals than male adrenals (c.f., Fig. 4B). Consistent with our hypothesis, adrenals collected from stressed male mice displayed lower PER2::LUC amplitude and larger responses to ACTH than adrenals collected from non-stressed male mice (Fig. 5B and C). Further, adrenals from females that received testosterone pellets displayed larger PER2::LUC amplitude prior to the ACTH pulse (Fig. 5A) and smaller responses to ACTH relative to surgical controls (Fig. 5B and C). As in our previous experiment, there was an inverse relationship between the magnitude of ACTH-induced responses and PER2::LUC amplitude prior to the pulse (resetting $-r=-0.43, P<0.005$; potentiation $-r=-0.69$, $P<0.0001)$. Collectively, these results suggest that the state of the adrenal clock gates the response to ACTH, which may contribute to sex differences in glucocorticoid release and stress reactivity.

\section{Discussion}

The present results demonstrate that exposure to stress in vivo influences the intrinsic expression of molecular rhythms in the adrenal gland in a sexually dimorphic manner. We also find a pronounced sex difference in the response of the adrenal clock to ACTH in vitro, which complements previous work demonstrating that female adrenals release more glucocorticoids when stimulated with ACTH in vitro (Kitay 1961b). Further, we provide evidence that ACTH responsiveness is gated by the amplitude of adrenal PER2::LUC rhythms, which reveals that there is an inverse relationship between the state of the adrenal clock and its response to ACTH stimulation. Collectively, these results suggest that there is a reciprocal interaction between the adrenal clock and its response to stress that is markedly influenced by sex. The sexually dimorphic function of the molecular clock in the adrenal may contribute to sex differences in stress reactivity given its role in gating glucocorticoid release (Son et al. 2011, Leliavski et al. 2014). Thus, long-term consequences of stress-induced changes in the adrenal clock warrant further study in both sexes.

Although the adrenal clock has been recognized for more than 50 years (Ungar \& Halberg 1962, Andrews \& Folk 1964), there have been only a handful of studies examining how exposure to stress alters its rhythms (Bartlang et al. 2014, Razzoli et al. 2014, Tahara et al. 2015, Engeland et al. 2016). We demonstrate here that chronic 
stress influences the intrinsic function of the adrenal clock in a sex- and stressor-dependent manner. Chronic restraint stress decreases PER2::LUC amplitude in both sexes, whereas FC+SPS increases PER2::LUC amplitude only in females. Opposite effects of these two stress procedures on adrenal clock function are noteworthy because these two stress procedures are used to model different stress-related disorders (Jankord \& Herman 2008, Yamamoto et al. 2009). Thus, differential effects of chronic restraint vs FC+SPS on adrenal clock function could reflect distinct cellular responses at the level of the adrenal and/or higher-order tissues. With this in mind, it is interesting that the effect of chronic restraint stress observed here is distinct from that commonly observed in other tissues of the brain and body (Oster et al. 2017). In most tissues examined to date, clock gene expression is increased by glucocorticoid signaling and/or stress (Oster et al. 2017), although stressor- and tissue-specific effects are not without precedent (Al-Safadi et al. 2014). Changes in tissues upstream of the adrenal could contribute to the resetting effects of stress observed in the adrenal gland here and in previous studies, which may be sensitive to the time of day stress is experienced (Jiang et al. 2011, Bartlang et al. 2014, Razzoli et al. 2014, Tahara et al. 2015, Engeland et al. 2016). Thus, future work is needed to test the potential role of higher-order tissues in mediating effects of stress on adrenal clock function, which should include consideration of the response to different stressors at varying times of day. Likewise, it will be of interest to assess how stress-induced changes in the state of the adrenal clock influence the function of other tissues, especially given evidence that the adrenal regulates molecular rhythms of other clock tissues in the circadian system (Balsalobre et al. 2000, Oishi et al. 2005, Segall et al. 2006, Reddy et al. 2007, Son et al. 2008, Kiessling et al. 2010, Pezuk et al. 2012).

The molecular clock gates approximately $10 \%$ of adrenal transcripts (Oster et al. 2006a, Son et al. 2008), including steroid acute regulatory protein (StAR) and the melanocortin 2 receptor (MC2R). Although mechanistic connections between the molecular clock and adrenal function are not fully understood, previous work suggests that the clock regulates glucocorticoid release under both basal and stressed conditions (Son et al. 2011, Leliavski et al. 2014). Further, results from mouse models lacking specific components of the molecular clock suggest that negative elements suppress glucocorticoid release (Dallmann et al. 2006, Chung et al. 2017), while positive elements elevate it (Kennaway et al. 2006, Leliavski et al. 2014). However, other studies using germ-line knockout strategies suggest that this model may be too simplistic (c.f., Pilorz et al. 2009, Yang et al. 2009). It also remains unclear precisely how the local adrenal clock contributes to glucocorticoid release. Abrogation of Bmal1 function in $M c 2 r$-expressing adrenal cells abolishes circadian corticosterone rhythms (Son et al. 2008). However, a recent study using a different strategy to eliminate Bmal1 in adrenocortical cells found no significant change in glucocorticoid release under basal or stressed conditions (Dumbell et al. 2016). Thus, the balance of work conducted so far indicates that the molecular clock regulates adrenal function, but understanding the nature and location of stress-circadian interactions requires further study. Our work suggests that novel insights into circadian regulation of adrenal function may be achieved by including females in molecular studies of the adrenal clock.

To our knowledge, this is the first study to examine the effects of sex on the response of the adrenal clock to stress, even though it is well known that glucocorticoid release is modulated by both sex and molecular clock function. Across species, sex differences in basal and stress-induced glucocorticoid release are well established (Goel et al. 2014). Relative to males, female mice display glucocorticoid rhythms with elevated release throughout the daytime (Feillet et al. 2016). The intrinsic function of the adrenal clock likewise displays a marked sexual dimorphism (Kuljis et al. 2013, Kloehn et al. 2016), as replicated here in our non-stressed controls. Although the molecular clock of the adrenal has only been extensively studied in males, one study suggests that the female adrenal in vivo displays lower Per1 levels and higher amplitude rhythms of the repressor Rev$e r b \alpha$ relative to the male adrenal (Feillet et al. 2016). Sex differences in circadian gene expression suggest that the clock-controlled function of the adrenal will be sexually dimorphic. Indeed, female mice in vivo display higher expression of Star and $\mathrm{Mc} 2 r$ in the adrenal (Feillet et al. 2016), and the latter may contribute to the sex difference in АCTH responsiveness that we observe here. Full understanding of sex differences in the adrenal clock awaits comprehensive mapping of the circadian landscape in both sexes (Dickmeis et al. 2013). In particular, how different components of the molecular clock intersect with adrenal signaling pathways in males and females should be examined further.

Although our understanding of sex differences in the adrenal clock remains incomplete, our present results indicate that its function can be modulated by stress in sexually dimorphic manner. The response of the adrenal clock to chronic restraint stress was qualitatively similar in both sexes, but differed quantitatively in male and 
female adrenals. Specifically, male adrenals displayed a larger reduction in amplitude after chronic restraint stress than female adrenals, but the effect in female adrenals was longer lasting. Even more striking was the sex-specific response to the FC+SPS procedure, which has been developed as a mouse model of posttraumatic stress disorder (Wang et al. 2012, Perrine et al. 2016). After exposure to FC+SPS, only female adrenals displayed an increase in adrenal amplitude, which again was a longlasting effect. We find this sexually dimorphic response noteworthy given the sex difference in the incidence of PTSD (Kessler et al. 1995, Brewin et al. 2000, Breslau 2002) and the association of PTSD in some patients with blunted basal glucocorticoid release and reduced sensitivity to ACTH (Heim et al. 2001, Kanter et al. 2001)

With this set of observations in mind, we feel one of the most interesting findings of the present work is the inverse relationship between adrenal PER2::LUC amplitude and the response of the adrenal clock to ACTH. Consistent with previous work (Yoder et al. 2014), we find that the ACTH response of the adrenal clock is phase dependent, with larger magnitude phase shifts during subjective night than subjective morning. This phasing complements the rhythm in ACTHinduced glucocorticoid release (Dallman et al. 1978, Kaneko et al. 1981). These two ACTH response rhythms persist ex vivo driven by the adrenal clock (Oster et al. 2006b, Yoder et al. 2014). Complementing previous work demonstrating that female adrenals release more glucocorticoids when stimulated with ACTH (Kitay 1961b), we find that molecular clock responses to ACTH are likewise greater in female adrenals. We find it interesting that ACTH increased PER2::LUC amplitude in vitro, whereas repeated restraint stress decreased PER2::LUC amplitude in vivo. This discrepancy in results likely reflects a difference in the nature of the stimulation. One possibility is that these results reflect a difference in the response of the adrenal clock to acute vs chronic stress. Consistent with this idea, a single episode of restraint stress produced a slight increase in PER2::LUC amplitude (Fig. 2D), but this effect was not statistically significant. Another possibility is that systemic factors modulate the response of the adrenal clock in vivo that are absent in vitro.

The results of our final experiment suggest that ACTH responsiveness is suppressed by increased PER2::LUC amplitude, which likely involves changes in the molecular control of MC2R and/or StAR expression
(Oster et al. 2006a, Son et al. 2008). Thus, the lower PER2::LUC amplitude displayed by female adrenals may contribute to their greater responses to ACTH and stress. On the other hand, the opposite relationship cannot be excluded presently. Thus, it remains possible that high glucocorticoid release in females suppresses Per expression in the adrenal (Dickmeis et al. 2013). Potential evidence against this alternative hypothesis stems from our findings that stress does not invariably decrease PER2::LUC amplitude. Chronic restraint stress did decrease amplitude, but acute restraint stress did not, even when adrenals were collected immediately after the procedure. Further, we find that the effect of stress on PER2::LUC amplitude is procedure specific. Unlike the decreases observed after chronic restraint stress, FC+SPS increased the amplitude and precision of adrenal PER2::LUC rhythms. Differential responses were elicited even though manipulations used in these two stress procedures can increase corticosterone release (Kelley et al. 2009, Jeong et al. 2013, Laukova et al. 2014). The mechanistic basis of this stressor-specific response warrants further research, especially as it pertains to sex differences in the response of the adrenal clock and longterm changes in physiology.

In summary, we demonstrate that stress influences molecular rhythms in the adrenal clock in a sexually dimorphic manner. We also provide evidence that the molecular state of the adrenal clock can regulate its sensitivity to ACTH. Stress-induced changes in adrenal clock function would be expected to have widespread effects on both circadian and non-circadian processes. Thus, it will be important to assess the long-term consequences of stress-related changes in adrenal clock function, especially in females for whom the effects appear to be long lasting. Future challenges are to identify the mechanisms of stress-clock crosstalk and how this machinery is influenced by sex. Further work is also required to define the precise roles of the different tissue clocks in regulating glucocorticoid release, especially given that the adrenal clock is not absolutely required under all conditions (Son et al. 2008, Dumbell et al. 2016). Lastly, it remains important to determine if changes in the function of the adrenal clock and/or other tissue clocks directly contribute to stress-related pathology. Our work highlights that the effect of stress on adrenal clock function depends on the stressor used, and thus comparing the effects of different stress models may provide insight into these important issues. 


\section{Declaration of interest}

The authors declare that there is no conflict of interest that could be perceived as prejudicing the impartiality of the research reported.

\section{Funding}

This work was supported by a Regular Research Grant from Marquette University. I K and M B were supported by the Marquette Undergraduate Summer Research Program.

\section{Author contribution statement}

M S, M B, C K, D W, I K, S P and J A E conducted experiments. M S, M B, C K, D W, I K, S P, M R G and J A E analyzed the data. P J G, M R G and J A E designed the experiments. J A E wrote the manuscript with the input of all authors.

\section{Acknowledgements}

The authors would like to thank Adriano Dellapolla, Claire Klement and Harshida Pancholi for their assistance in conducting these studies.

\section{References}

Al-Safadi S, Al-Safadi A, Branchaud M, Rutherford S, Dayanandan A, Robinson B \& Amir S 2014 Stress-induced changes in the expression of the clock protein PERIOD1 in the rat limbic forebrain and hypothalamus: Role of stress type, time of day, and predictability. PLOS ONE 9 e111166. (https://doi.org/10.1371/journal. pone.0111166)

Andrews RV \& Folk GE Jr 1964 Circadian metabolic patterns in cultured hamster adrenal glands. Comparative Biochemistry and Physiology 11 393-409. (https://doi.org/10.1016/0010-406X(64)90006-4)

Balsalobre A, Brown SA, Marcacci L, Tronche F, Kellendonk C, Reichardt HM, Schutz G \& Schibler U 2000 Resetting of circadian time in peripheral tissues by glucocorticoid signaling. Science $\mathbf{2 8 9}$ 2344-2347. (https://doi.org/10.1126/science.289.5488.2344)

Bangasser DA \& Valentino RJ 2014 Sex differences in stress-related psychiatric disorders: neurobiological perspectives. Frontiers in Neuroendocrinology 35 303-319. (https://doi.org/10.1016/j. yfrne.2014.03.008)

Bartlang MS, Savelyev SA, Johansson AS, Reber SO, Helfrich-Forster C \& Lundkvist GBS 2014 Repeated psychosocial stress at night, but not day, affects the central molecular clock. Chronobiology International 31 996-1007. (https://doi.org/10.3109/07420528.2014.940085)

Boksa P \& Livett BG 1984 Desensitization to nicotinic cholinergic agonists and $\mathrm{K}+$, agents that stimulate catecholamine secretion in isolated adrenal chromaffin cells. Journal of Neurochemistry 42 607-617. (https://doi.org/10.1111/j.1471-4159.1984.tb02726.x)

Boland EM \& Ross RJ 2015 Recent advances in the study of sleep in the anxiety disorders, obsessive-compulsive disorder, and posttraumatic stress disorder. Psychiatric Clinics of North America 38 761-776. (https://doi.org/10.1016/j.psc.2015.07.005)

Breslau N 2002 Gender differences in trauma and posttraumatic stress disorder. Journal of Gender-Specific Medicine 5 34-40.

Brewin CR, Andrews B \& Valentine JD 2000 Meta-analysis of risk factors for posttraumatic stress disorder in trauma-exposed adults. Journal of Consulting and Clinical Psychology 68 748-766. (https://doi. org/10.1037/0022-006X.68.5.748)
Buhr ED \& Takahashi JS 2013 Molecular components of the mammalian circadian clock. Handbook of Experimental Pharmacology 3-27. (https://doi.org/10.1007/978-3-642-25950-0_1)

Chung S, Lee EJ, Cha HK, Kim J, Kim D, Son GH \& Kim K 2017 Cooperative roles of the suprachiasmatic nucleus central clock and the adrenal clock in controlling circadian glucocorticoid rhythm. Scientific Reports 7 46404. (https://doi.org/10.1038/srep46404)

Dallman MF, Engeland WC, Rose JC, Wilkinson CW, Shinsako J \& Siedenburg F 1978 Nycthemeral rhythm in adrenal responsiveness to ACTH. American Journal of Physiology 235 R210-R218. (https://doi. org/10.1152/ajpregu.1978.235.5.R210)

Dallmann R, Touma C, Palme R, Albrecht U \& Steinlechner S 2006 Impaired daily glucocorticoid rhythm in Per1 (Brd) mice. Journal of Comparative Physiology A: Neuroethology, Sensory, Neural, and Behavioral Physiology 192 769-775. (https://doi.org/10.1007/s00359-006-0114-9)

Daskalakis NP, Yehuda R \& Diamond DM 2013 Animal models in translational studies of PTSD. Psychoneuroendocrinology 38 1895-1911. (https://doi.org/10.1016/j.psyneuen.2013.06.006)

Davidson AJ, Castanon-Cervantes O, Leise TL, Molyneux PC \& Harrington ME 2009 Visualizing jet lag in the mouse suprachiasmatic nucleus and peripheral circadian timing system. European Journal of Neuroscience 29 171-180. (https://doi. org/10.1111/j.1460-9568.2008.06534.x)

Dellapolla A, Kloehn I, Pancholi H, Callif B, Wertz D, Rohr KE, Hurley MM, Baker KM, Hattar S, Gilmartin MR, et al. 2017 Long days enhance recognition memory and increase insulin-like growth factor 2 in the hippocampus. Scientific Reports 7 3925. (https://doi. org/10.1038/s41598-017-03896-2)

Demas GE \& Nelson RJ 1998 Short-day enhancement of immune function is independent of steroid hormones in deer mice (Peromyscus maniculatus). Journal of Comparative Physiology B 168 419-426. (https://doi.org/10.1007/s003600050161)

Dickmeis T, Weger BD \& Weger M 2013 The circadian clock and glucocorticoids - interactions across many time scales. Molecular and Cellular Endocrinology 380 2-15. (https://doi.org/10.1016/j. mce.2013.05.012)

Dumbell R, Leliavski A, Matveeva O, Blaum C, Tsang AH \& Oster H 2016 Dissociation of molecular and endocrine circadian rhythms in male mice lacking Bmal1 in the adrenal cortex. Endocrinology 157 4222-4233. (https://doi.org/10.1210/en.2016-1330)

Engeland WC, Yoder JM, Karsten CA \& Kofuji P 2016 Phase-dependent shifting of the adrenal clock by acute stress-induced ACTH. Frontiers in Endocrinology 7 81. (https://doi.org/10.3389/fendo.2016.00081)

Feillet C, Guerin S, Lonchampt M, Dacquet C, Gustafsson JA, Delaunay F \& Teboul M 2016 Sexual dimorphism in circadian physiology is altered in LXRalpha deficient mice. PLOS ONE 11 e0150665. (https://doi.org/10.1371/journal.pone.0150665)

Goel N, Workman JL, Lee TT, Innala L \& Viau V 2014 Sex differences in the HPA axis. Comprehensive Physiology 4 1121-1155. (https://doi. org/10.1002/cphy.c130054)

Heim C, Newport DJ, Bonsall R, Miller AH \& Nemeroff CB 2001 Altered pituitary-adrenal axis responses to provocative challenge tests in adult survivors of childhood abuse. American Journal of Psychiatry 158 575-581. (https://doi.org/10.1176/appi.ajp.158.4.575)

Ishida A, Mutoh T, Ueyama T, Bando H, Masubuchi S, Nakahara D, Tsujimoto G \& Okamura H 2005 Light activates the adrenal gland: Timing of gene expression and glucocorticoid release. Cell Metabolism 2 297-307. (https://doi.org/10.1016/j.cmet.2005.09.009)

Jankord R \& Herman JP 2008 Limbic regulation of hypothalamopituitary-adrenocortical function during acute and chronic stress. Annals of the New York Academy of Sciences 1148 64-73. (https://doi. org/10.1196/annals.1410.012)

Jeong JY, Lee DH \& Kang SS 2013 Effects of chronic restraint stress on body weight, food intake, and hypothalamic gene expressions in 
mice. Endocrinology and Metabolism 28 288-296. (https://doi. org/10.3803/EnM.2013.28.4.288)

Jiang WG, Li SX, Zhou SJ, Sun Y, Shi J \& Lu L 2011 Chronic unpredictable stress induces a reversible change of PER2 rhythm in the suprachiasmatic nucleus. Brain Research 1399 25-32. (https://doi. org/10.1016/j.brainres.2011.05.001)

Kaneko M, Kaneko K, Shinsako J \& Dallman MF 1981 Adrenal sensitivity to adrenocorticotropin varies diurnally. Endocrinology 109 70-75. (https://doi.org/10.1210/endo-109-1-70)

Kanter ED, Wilkinson CW, Radant AD, Petrie EC, Dobie DJ, McFall ME, Peskind ER \& Raskind MA 2001 Glucocorticoid feedback sensitivity and adrenocortical responsiveness in posttraumatic stress disorder. Biological Psychiatry 50 238-245. (https://doi.org/10.1016/S00063223(01)01158-1)

Kelley JB, Balda MA, Anderson KL \& Itzhak Y 2009 Impairments in fear conditioning in mice lacking the nNOS gene. Learning and Memory 16 371-378. (https://doi.org/10.1101/lm.1329209)

Kennaway DJ, Owens JA, Voultsios A \& Varcoe TJ 2006 Functional central rhythmicity and light entrainment, but not liver and muscle rhythmicity, are Clock independent. American Journal of Physiology 291 R1172-R1180. (https://doi.org/10.1152/ajpcell.00195.2006)

Kessler RC, Sonnega A, Bromet E, Hughes M \& Nelson CB 1995 Posttraumatic stress disorder in the national comorbidity survey. Archives of General Psychiatry 52 1048-1060. (https://doi.org/10.1001/ archpsyc.1995.03950240066012)

Kiessling S, Eichele G \& Oster H 2010 Adrenal glucocorticoids have a key role in circadian resynchronization in a mouse model of jet lag. Journal of Clinical Investigation 120 2600-2609. (https://doi. org/10.1172/JCI41192)

Kitay JI 1961a Enhancement of steroidogenesis by rat adrenal slices in vitro with estradiol-17-beta. Nature 192 358-359. (https://doi. org/10.1038/192358b0)

Kitay JI $1961 b$ Sex differences in adrenal cortical secretion in the rat. Endocrinology 68 818-824. (https://doi.org/10.1210/endo-68-5-818)

Kloehn I, Pillai SB, Officer L, Klement C, Gasser PJ \& Evans JA 2016 Sexual differentiation of circadian clock function in the adrenal gland. Endocrinology 157 1895-1904. (https://doi.org/10.1210/ en.2015-1968)

Kronfeld-Schor N \& Einat H 2012 Circadian rhythms and depression: Human psychopathology and animal models. Neuropharmacology 62 101-114. (https://doi.org/10.1016/j. neuropharm.2011.08.020)

Kuljis DA, Loh DH, Truong D, Vosko AM, Ong ML, McClusky R, Arnold AP \& Colwell CS 2013 Gonadal- and sex-chromosomedependent sex differences in the circadian system. Endocrinology 154 1501-1512. (https://doi.org/10.1210/en.2012-1921)

Laukova M, Alaluf LG, Serova LI, Arango V \& Sabban EL 2014 Early intervention with intranasal NPY prevents single prolonged stresstriggered impairments in hypothalamus and ventral hippocampus in male rats. Endocrinology 155 3920-3933. (https://doi.org/10.1210/ en.2014-1192)

Leliavski A, Shostak A, Husse J \& Oster H 2014 Impaired glucocorticoid production and response to stress in Arntl-deficient male mice. Endocrinology 155 133-142. (https://doi.org/10.1210/en.2013-1531)

McQuade JM, Tamashiro KL, Wood GE, Herman JP, McEwen BS, Sakai RR, Zhang J \& Xu M 2006 Deficient hippocampal c-fos expression results in reduced anxiety and altered response to chronic stress in female mice. Neuroscience Letters 403 125-130. (https://doi. org/10.1016/j.neulet.2006.04.022)

Mohawk JA, Green CB \& Takahashi JS 2012 Central and peripheral circadian clocks in mammals. Annual Review of Neuroscience $\mathbf{3 5}$ 445-462. (https://doi.org/10.1146/annurev-neuro-060909-153128)

Nicolaides NC, Charmandari E, Kino T \& Chrousos GP 2017 Stressrelated and circadian secretion and target tissue actions of glucocorticoids: Impact on health. Frontiers in Endocrinology 870. (https://doi.org/10.3389/fendo.2017.00070)
Oishi K, Amagai N, Shirai H, Kadota K, Ohkura N \& Ishida N 2005 Genome-wide expression analysis reveals 100 adrenal glanddependent circadian genes in the mouse liver. DNA Research 12 191-202. (https://doi.org/10.1093/dnares/dsi003)

Oster H, Damerow S, Hut RA \& Eichele G $2006 a$ Transcriptional profiling in the adrenal gland reveals circadian regulation of hormone biosynthesis genes and nucleosome assembly genes. Journal of Biological Rhythms 21 350-361. (https://doi. org/10.1177/0748730406293053)

Oster H, Damerow S, Kiessling S, Jakubcakova V, Abraham D, Tian J, Hoffmann MW \& Eichele G 2006b The circadian rhythm of glucocorticoids is regulated by a gating mechanism residing in the adrenal cortical clock. Cell Metabolism 4 163-173. (https://doi. org/10.1016/j.cmet.2006.07.002)

Oster H, Challet E, Ott V, Arvat E, Ronald de Kloet E, Dijk DJ, Lightman S, Vgontzas A \& Van Cauter E 2017 The functional and clinical significance of the 24-hour rhythm of circulating glucocorticoids. Endocrine Reviews 38 3-45. (https://doi.org/10.1210/ er.2017.38.issue-2.edboard)

Perrine SA, Eagle AL, George SA, Mulo K, Kohler RJ, Gerard J, Harutyunyan A, Hool SM, Susick LL, Schneider BL, et al. 2016 Severe, multimodal stress exposure induces PTSD-like characteristics in a mouse model of single prolonged stress. Behavioural Brain Research 303 228-237. (https://doi.org/10.1016/j.bbr.2016.01.056)

Pezuk P, Mohawk JA, Wang LA \& Menaker M 2012 Glucocorticoids as entraining signals for peripheral circadian oscillators. Endocrinology 153 4775-4783. (https://doi.org/10.1210/en.2012-1486)

Pilorz V, Steinlechner S \& Oster H 2009 Age and oestrus cycle-related changes in glucocorticoid excretion and wheel-running activity in female mice carrying mutations in the circadian clock genes Per1 and Per2. Physiology and Behavior 96 57-63. (https://doi. org/10.1016/j.physbeh.2008.08.010)

Razzoli M, Karsten C, Yoder JM, Bartolomucci A \& Engeland WC 2014 Chronic subordination stress phase advances adrenal and anterior pituitary clock gene rhythms. American Journal of Physiology $\mathbf{3 0 7}$ R198-R205. (https://doi.org/10.1152/ajpregu.00101.2014)

Reddy AB, Maywood ES, Karp NA, King VM, Inoue Y, Gonzalez FJ, Lilley KS, Kyriacou CP \& Hastings MH 2007 Glucocorticoid signaling synchronizes the liver circadian transcriptome. Hepatology $\mathbf{4 5}$ 1478-1488. (https://doi.org/10.1002/hep.21571)

Rilianawati, Kero J, Paukku T \& Huhtaniemi I 2000 Long-term testosterone treatment prevents gonadal and adrenal tumorigenesis of mice transgenic for the mouse inhibin-alpha subunit promoter/ simian virus 40 T-antigen fusion gene. Journal of Endocrinology 166 77-85. (https://doi.org/10.1677/joe.0.1660077)

Segall LA, Perrin JS, Walker CD, Stewart J \& Amir S 2006 Glucocorticoid rhythms control the rhythm of expression of the clock protein, PERIOD2, in oval nucleus of the bed nucleus of the stria terminalis and central nucleus of the amygdala in rats. Neuroscience $\mathbf{1 4 0}$ 753-757. (https://doi.org/10.1016/j.neuroscience.2006.03.037)

Son GH, Chung S, Choe HK, Kim HD, Baik SM, Lee H, Lee HW, Choi S, Sun W, Kim H, et al. 2008 Adrenal peripheral clock controls the autonomous circadian rhythm of glucocorticoid by causing rhythmic steroid production. PNAS 105 20970-20975. (https://doi. org/10.1073/pnas.0806962106)

Son GH, Chung S \& Kim K 2011 The adrenal peripheral clock: Glucocorticoid and the circadian timing system. Frontiers in Neuroendocrinology 32 451-465. (https://doi.org/10.1016/j. yfrne.2011.07.003)

Tahara Y, Shiraishi T, Kikuchi Y, Haraguchi A, Kuriki D, Sasaki H, Motohashi H, Sakai T \& Shibata S 2015 Entrainment of the mouse circadian clock by sub-acute physical and psychological stress. Scientific Reports 5 11417. (https://doi.org/10.1038/srep11417)

Ungar F \& Halberg F 1962 Circadian rhythm in the in vitro response of mouse adrenal to adrenocorticotropic hormone. Science $\mathbf{1 3 7}$ 1058-1060. (https://doi.org/10.1126/science.137.3535.1058) 
Wang H, Zuo D, He B, Qiao F, Zhao M \& Wu Y 2012 Conditioned fear stress combined with single-prolonged stress: A new PTSD mouse model. Neuroscience Research 73 142-152. (https://doi.org/10.1016/j. neures.2012.03.003)

Yamamoto S, Morinobu S, Takei S, Fuchikami M, Matsuki A, Yamawaki S \& Liberzon I 2009 Single prolonged stress: Toward an animal model of posttraumatic stress disorder. Depression and Anxiety 26 1110-1117. (https://doi.org/10.1002/da.20629)

Yang S, Liu A, Weidenhammer A, Cooksey RC, McClain D, Kim MK, Aguilera G, Abel ED \& Chung JH 2009 The role of mPer2 clock gene in glucocorticoid and feeding rhythms. Endocrinology $\mathbf{1 5 0}$ 2153-2160. (https://doi.org/10.1210/en.2008-0705)

Yoder JM, Brandeland M \& Engeland WC 2014 Phase-dependent resetting of the adrenal clock by ACTH in vitro. American Journal of Physiology 306 R387-R393.

Yoo SH, Yamazaki S, Lowrey PL, Shimomura K, Ko CH, Buhr ED, Siepka SM, Hong HK, Oh WJ, Yoo OJ, et al. 2004

PERIOD2::LUCIFERASE real-time reporting of circadian dynamics reveals persistent circadian oscillations in mouse peripheral tissues. PNAS 101 5339-5346. (https://doi.org/10.1073/pnas.0308709101)

Received in final form 29 November 2017

Accepted 4 December 2017 